\title{
Altmetria de artigos de periódicos brasileiros de acesso aberto na ScienceOpen: uma análise das razões de menções
}

\author{
Altmetrics of open access brazilian journal articles in ScienceOpen: the \\ reason for mentions
}

\section{Altmétricas de los artículos en periódicos brasileños de acceso abierto en ScienceOpen: análisis de las razones de sus menciones}

\author{
Ronaldo Ferreira de Araujo | ronaldfa@gmail.com \\ Universidade Federal de Alagoas, Instituto de Ciências Humanas, Comunicação e Artes, Curso de \\ Biblioteconomia. Maceió, Brasil.
}

Marlene Oliveira | marlene@eci.ufmg.br

Universidade Federal de Minas Gerais, Departamento de Organização e Tratamento da Informação, Programa de Pós-Graduação em Gestão e Organização do Conhecimento. Belo Horizonte, Brasil.

Elaine Rosangela de Oliveira Lucas | lanilucas@gmail.com

Universidade do Estado de Santa Catarina, Departamento de Biblioteconomia, Programa de Pós-Graduação em Gestao da Informação. Florianópolis, Brasil.

\section{Resumo}

Procura contribuir com debate acerca dos estudos qualitativos no campo da altmetria aplicando a análise de menções de Araújo e Furnival (2016) a um conjunto de artigos de periódicos de acesso aberto presentes na ScienceOpen da Coleção SciELO Brasil. Ao todo foram identificados 1.976 dados altmétricos de artigos publicados entre 2001 e 2015. A maioria dos dados gerados provém do Twitter $(68,9 \%)$, seguido do Facebook $(18,4 \%)$ e do Mendeley (12,3\%). As menções estão distribuídas quanto ao seu teor informativo $(55,1 \%)$ e conversacional (44,9\%), tendo como razão predominante a simples disseminação (57,5\%), seguido de breve comentário de cunho explicativo de parte de seu texto $(17,4 \%)$ e de narrativas exortativas, nas quais se utiliza do estudo para sensibilizar práticas e ações (15,8\%). Não foram registradas mensagens que criticam ou problematizam os artigos. Dado aos poucos estudos sobre o tema abordado torna-se necessário investir em propostas que considerem a análise das menções com vistas a entender as nuances da circulação da informação científica na web social e suas perspectivas.

Palavras-chave: altmetria; análise de menções; acesso aberto; ScienceOpen. 


\begin{abstract}
This study aims to contribute to a debate about qualitative studies in the field of altmetrics applying the analysis of mentions of Araújo and Furnival (2016) to a set of articles of open access journals present in ScienceOpen of the SciELO Brazil Collection. We identified 1,976 altmetric data from articles published between 2001 and 2015. Most of the data generated came from Twitter (68.9\%), followed by Facebook (18.4\%) and Mendeley (12.3\%). The mentions are distributed according to their informative content (55.1\%) and conversational (44.9\%) aspects, with predominantly simple sharing (57.5\%), followed by explanatory comments (17.4\%), and of exortative narratives (15.8\%). Messages that criticize or problematize articles have not been registered. We conclude that due to the few studies on the subject it is necessary to invest in proposals that consider the analysis of the mentions to understand the nuances of the circulation of scientific information in the social web and its perspectives.
\end{abstract}

Keywords: altmetrics; mention analysis; open access; ScienceOpen.

\title{
Resumen
}

La presente comunicación busca contribuir con el debate acerca de los estudios cualitativos en el campo de las altmétricas, aplicando el análisis de menciones de Araújo y Furnival (2016) a un conjunto de artículos en periódicos de acceso abierto presentes en la ScienceOpen de la Colección SciELO Brasil. En total se identificaron 1.976 datos altmétricos de artículos publicados entre 2001 y 2015. La mayoría de los datos generados provienen de Twitter (68,9\%), seguido de Facebook (18,4\%) y de Mendeley (12,3\%). Las menciones están distribuidas en cuanto a sus contenidos informativo $(55,1 \%)$ y conversacional $(44,9 \%)$. Ademas, tienem como razón predominante la simple diseminación (57,5\%), seguido de breve comentario de cuño explicativo de parte de su texto $(17,4 \%)$ y de narrativas 'exortativas', en las que se utiliza del estudio para sensibilizar prácticas y acciones (15,8\%). No se registraron mensajes que critican o problematizan los artículos. A los pocos estudios sobre el tema abordado se hace necesario invertir en propuestas que consideren el análisis de las menciones con miras a entender los matices de la circulación de la información científica en la web social y sus perspectivas.

Palabras clave: Altmétricas; análisis de menciones; acceso abierto; ScienceOpen.

\section{Introdução}

Os estudos métricos da informação em ciência e tecnologia indicam que artigos publicados em acesso aberto costumam ter vantagens em seus indicadores tradicionais de avaliação como nos estudos bibliométricos de análise de citação. O mesmo comportamento parece ser válido quando se trata de indicadores alternativos, aos quais assim como os primeiros, carecem de estudos qualitativos que ofereçam condições de aferir sobre as formas e contextos de uso em que textos científicos circulam nas mídias sociais.

Ainda que pesquisas informétricas desenvolvidas por meio de aplicações matemáticas e estatísticas consistentes sejam capazes de gerar indicadores quantitativos eficientes para inúmeras atividades de gestão e servir de apoio á tomada de decisões, é fundamental que elas sejam pensadas de forma conjugada com abordagens qualitativas.

A análise de razões das citações, por exemplo, é uma perspectiva de estudo que busca compreender os fenômenos de produção e uso da informação científica registrada, ao considerar possíveis fatores objetivos e subjetivos que motivam as citações conforme afirmam Silveira, Caregnato e Bufrem¹. De igual modo, surgem no emergente campo da altmetria, estudos que investigam os tipos de interações que os artigos científicos recebem nas mídias sociais a fim de caracterizar suas menções e compreender suas implicações para a comunicação científica.

De acordo com Gouveia ${ }^{2}$ a altmetria pode ser vista como o uso de dados cibermétricos para análises cientométricas e para o autor da mesma forma que as citações, as menções em mídias sociais como 
mensagens no Twitter poderiam indicar um diálogo ou interesse numa obra, mas não atestariam a qualidade do que está sendo dito.

Assim, uma abordagem que levasse em consideração o contexto das interações, o conteúdo das mensagens poderia contribuir com uma reflexão sobre o que os valores altmétricos tem a dizer sobre determinada publicação, inclusive, tendo em vista que nas palavras de Gouveia ${ }^{2}$ um olhar sobre os comentários seria uma oportunidade de se detectar precocemente o interesse ou polêmica pelos resultados apresentados. O autor acrescenta ainda que esta prática seria estratégica para ações de comunicação do pesquisador ou instituição no debate que venha a se seguir com a imprensa e a sociedade como um todo

A pesquisa de Araújo e Furnival ${ }^{3}$ foi desenvolvida nessa perspectiva. Por meio de um estudo inspirado no trabalho de Nelhans e Lorentzen ${ }^{4}$, os autores buscaram compreender a formação de colégios virtuais que sustentam as métricas alternativas de determinada produção científica, ou seja, identificar quem eram os usuários que mencionavam artigos nas mídias sociais e principalmente verificar como esses artigos eram mencionados.

Assim Araújo e Furnival $^{3}$ trabalharam com seis categorias para analisar como artigos científicos são mencionados no Twitter e Facebook, por meio de um procedimento qualitativo de análise de conteúdo, adequado para análise do contexto de mensagens textuais.

Este tipo de verificação contribui na construção de inferências replicáveis e válidas de textos aos contextos do seu uso. As categorias investigadas foram as seguintes:

(a) compartilhamento (repasse): na qual há uma cópia do URL do artigo com uma breve síntese do conteúdo, semelhante ao seu título ou resumo;

(b) explicativo: procura detalhar com maior ênfase os resultados do estudo. Apresenta dados do estudo;

(c) conclusivo: afere credibilidade científica do estudo; qualifica o estudo como incontestável (devido ao método, tamanho da amostra, etc); se utiliza do estudo para refutar outras teorias;

(d) argumentativo: argumenta/discute um ponto do artigo, geralmente com tom energicamente a favor da abordagem do estudo. Lista este estudo entre outros semelhantes;

(e) exortativo: Se utiliza do estudo para sensibilizar práticas e ações;

(f) problematizante: mensagens de crítica e/ou desqualificação do estudo, quanto a seus aspectos gerais, teóricos ou metodológicos.

A presente pesquisa procura contribuir com debate acerca dos estudos qualitativos no campo da altmetria aplicando a análise de menções de Araújo e Furnival ${ }^{3}$ a um conjunto de artigos de periódicos de acesso aberto presentes na ScienceOpen da Coleção SciELO Brasil.

\section{Material e Método}

Trata-se de um estudo exploratório de cunho descritivo com o intuito de avaliar o uso de métricas alternativas para artigos de periódicos brasileiros de acesso aberto por meio de uma abordagem qualitativa de análise.

O universo de pesquisa é composto com um conjunto de artigos de periódicos de acesso aberto presentes na ScienceOpen (www.scienceopen.com), plataforma de pesquisa e serviço de descoberta de livre acesso que promove a contextualização da pesquisa, permitindo aos usuários inserir comentários, fazer recomendações ou mesmo revisões de pares pós-publicação.

A ScienceOpen possui atualmente cerca de 28 milhões de registros que podem ser buscados com aplicação de alguns filtros como título, resumo, palavras-chave, autor, periódico, data de publicação, áreas de conhecimento e coleções. E seus resultados podem ser ordenados por relevância, classificação média, data de publicação, número de citação, leituras ou pontuação altmétrica. Esta última é gerada pelo serviço da Altmetric.com. 
As coleções da ScienceOpen são consideradas grupos temáticos ou institucionais de documentos de pesquisa revisados por pares, de uma variedade de editores e revistas. O objetivo principal das coleções da ScienceOpen é proporcionar um espaço comunitário para a criação, distribuição e avaliação de informações acadêmicas.

Para localização dos artigos de periódicos brasileiros a busca foi realizada na primeira semana de março de 2017, por meio da navegação junto à Coleção SciELO Brazili. Os artigos da coleção foram ordenados por maior valor altmétrico (Sort by Altmetric Score) e considerados, por meio de uma amostra intencional não probabilística, apenas os dez primeiros cujo idioma fosse o Português, os quais foram publicados entre 2001 e 2015 e podem ser observados no Quadro 1, com indicação de seus autores, título e periódico.

Quadro 1 - Artigos com maior pontuação altmétrica da Coleção Scielo no ScienceOpen

\begin{tabular}{|c|c|c|c|}
\hline Autores & Titulo & Periódico & Ano \\
\hline $\begin{array}{l}\text { C Weinberg; } \\
\text { T A Cordás; } \\
\text { P A Munoz }\end{array}$ & Santa Rosa de Lima: uma santa anoréxica na América Latina? & $\begin{array}{l}\text { Soc. de Psiq. Rio } \\
\text { Grande do Sul }\end{array}$ & 2005 \\
\hline $\begin{array}{l}\text { L Marchiori; } \\
\text { E R Filho; } \\
\text { T Matsuo }\end{array}$ & Hipertensão como fator associado à perda auditiva & Rev. Bras. Otor. & 2006 \\
\hline $\begin{array}{l}\text { M Mancini; } \\
\text { A Halpern }\end{array}$ & Tratamento Farmacológico da Obesidade & $\begin{array}{l}\text { Arq. Bras. Endoc. \& } \\
\text { Metab. }\end{array}$ & 2002 \\
\hline M Tellez & Você é tão inteligente! por que não estudou medicina? & Rev. Esc. Enf. USP & 2013 \\
\hline $\begin{array}{l}\text { M G Oliveira } \\
\text { Neto }\end{array}$ & $\begin{array}{l}\text { Entre o grotesco e o risível: o lugar da mulher negra na história } \\
\text { em quadrinhos no Brasil }\end{array}$ & Rev. Bras. Ci. Política & 2015 \\
\hline M Teles & Violações dos direitos humanos das mulheres na ditadura & Est. Feministas & 2015 \\
\hline F O Almeida & $\begin{array}{l}\text { A arqueologia dos fermentados: a etílica história dos Tupi- } \\
\text { Guarani }\end{array}$ & Est. Avanç. & 2015 \\
\hline $\begin{array}{l}\text { P Simões; } \\
\text { R Soares }\end{array}$ & $\begin{array}{l}\text { Efeitos do Programa Bolsa Família na fecundidade das } \\
\text { beneficiárias }\end{array}$ & Rev. Bras. de Econ. & 2012 \\
\hline P Freire & Carta de Paulo Freire aos professores & Est. Avanç. & 2001 \\
\hline $\begin{array}{l}\text { F Olmos; } \\
\text { W A G Silva; } \\
\text { C Albano }\end{array}$ & $\begin{array}{l}\text { Aves em oito áreas de Caatinga no Sul do Ceará e Oeste } \\
\text { de Pernambuco, nordeste do Brasil: composição, riqueza e } \\
\text { similaridade }\end{array}$ & $\begin{array}{l}\text { Papéis Avulsos de } \\
\text { Zoologia }\end{array}$ & 2005 \\
\hline
\end{tabular}

Fonte: dados da pesquisa (2017)

Os artigos forneceram 1.976 dados altmétricos. A amostra foi considerada suficiente à proposta da pesquisa de contribuir com uma abordagem qualitativa para os estudos de altmetria aplicando as categorias (compartilhamento; explicativo; conclusivo; argumentativo; exortativo; e problematizante) de análise de menções de Araújo e Furnival3.

i Disponível em: https://www.scienceopen.com/search\#collection/3f2fc474-1b89-4787-a968-69184d0cc2f9 


\section{Análise e discussão dos resultados}

Os artigos analisados foram publicados nas seguintes áreas: Ciências da Saúde (4), Ciências Sociais Aplicadas (3), Ciências Humanas (2) e Ciências Biológicas (1). Os quatro artigos com maior valor altmétrico são de revistas da área da Saúde. O resultado é similar ao do estudo de Costas, Zahedi e Wouters ${ }^{5}$ no qual as Ciências da Saúde também foi a área de conhecimento que obteve maior atenção online, com valores altmétricos superiores as demais.

O Gráfico 1 apresenta a distribuição dos valores altmétricos obtidos pelas publicações em cada uma das fontes nas quais foram mencionadas.

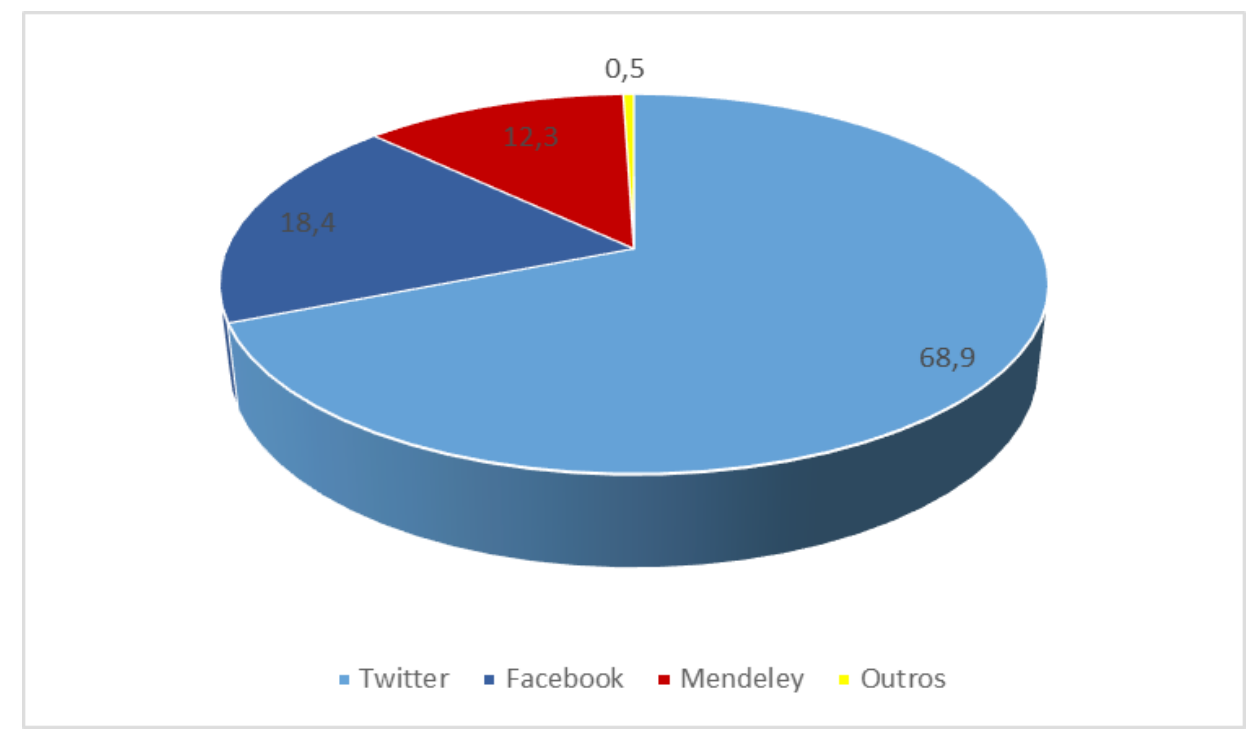

Gráfico 1 - Fontes altmétricas dos artigos analisados

Fonte: dados da pesquisa (2017)

Os resultados indicam que a maioria dos dados altmétricos gerados provêm do Twitter (68,9\%), seguido do Facebook (18,4\%), do Mendeley (12,3\%) e de outras fontes (0,5\%). A distribuição entre fontes tem comportamento similar com o resultado de outras pesquisas que utilizaram dados gerados pela Almetric. com, como nos estudos de Araújo e Furnival ${ }^{3}$, Alperin ${ }^{6}$, Boon e Foon ${ }^{7}$, e Costas, Zahedi e Wouters ${ }^{5}$, que apontam certa predileção pelo Twiiter por parte dos usuários que compartilham artigos científicos nas mídias sociais, seguido do Facebook e do Mendeley.

As práticas sociais que decorrem dos valores construídos e percebidos por usuários de mídias sociais são geralmente divididas entre aquelas de conteúdo e as de conversação. De acordo com Fragoso, Recuero e Amaral ${ }^{8}$ a primeira refere-se à relevância e influência das informações publicadas, na busca de levá-las a um determinado grupo e a segunda relaciona-se aquelas que visam a iniciar um diálogo, uma troca de informações, e por sua vez, espaço de conversação. A Tabela 1 apresenta a distribuição das menções nessas duas categorias relacionadas aos valores das mensagens postadas, seja em seu propósito informativo ou interacional.

Tabela 1 - Quantidade de Menções por tipo

\begin{tabular}{l|l|l}
\hline Tipo & Quantidade & \% \\
\hline Informativo & 1088 & 55,1 \\
\hline Conversacional & 888 & 44,9 \\
\hline Total & $\mathbf{1 9 7 6}$ & $\mathbf{1 0 0}$ \\
\hline
\end{tabular}

Fonte: dados da pesquisa (2017) 
Não foi identificado alguma disparidade acentuada entre os tipos de menções dos artigos uma vez que postagens relacionadas às práticas sociais de conteúdo e, portanto, informativas representam 55,1\%, indicativo de menções que divulgam os artigos; contra 44,9\% de postagens de conversação, ou seja, mensagens que mencionam outros usuários no conteúdo vinculado sobre o artigo, tendo um cunho interativo. Por fim, a Tabela 2 apresenta a distribuição das menções pelas categorias e, portanto, procuram contextualizar os valores altmétricos vinculando-os as suas possíveis razões.

Tabela 2 - Distribuição dos dados altmétricos por razões de menções

\begin{tabular}{l|l|l}
\hline Categorias & Valor & \% \\
\hline Compartilhamento & 1136 & 57,5 \\
\hline Explicativo & 344 & 17,4 \\
\hline Conclusivo & 168 & 8,5 \\
\hline Argumentativo & 16 & 0,8 \\
\hline Exortativo & 312 & 15,8 \\
\hline Problematizante & 0 & 0 \\
\hline Total & $\mathbf{1 9 7 6}$ & $\mathbf{1 0 0}$ \\
\hline
\end{tabular}

Fonte: dados da pesquisa (2017)

Quanto às categorias de razões das menções e que possivelmente motivaram a circulação dos artigos nas mídias sociais, têm-se como razão predominante a simples disseminação ou ato de compartilhar o artigo na rede (57,5\%), seguido de breve comentário de cunho explicativo de parte do artigo (17,4\%) e de narrativas exortativas, nas quais se utiliza do estudo para sensibilizar práticas e ações $(15,8 \%)$. A seguir estão as mensagens de teor conclusivo $(8,5 \%)$ e, em menor expressão, as de razões argumentativas (o,8\%). Não foram identificadas mensagens na categoria problematizante, que agruparia críticas ao artigo mencionado.

A análise da distribuição por categorias de menções é semelhante a encontrada na pesquisa de Araújo e Furnival ${ }^{3}$ que também obteve a razão de compartilhar ou repassar como sendo a mais representativa. Para os autores, este tipo de replicação exige menos comprometimento do usuário e poderia ser visto como "econômico" ou "apressado" no sentido que o usuário simplesmente quer divulgar a fonte original entre os participantes da sua rede. Este comportamento também pode ser interpretado como "neutro", pois ao compartilhar a fonte original sem comentá-la, não é possível identificar "em qual lado" do argumento, localiza-se quem o compartilha como já mencionado por Araújo e Furnival ${ }^{3}$.

As demais categorias (explicativo, conclusivo, argumentativo, exortativo) comportam mensagens que exigiram algum nível de produção de conteúdo em relação aos artigos por parte dos usuários. Além de compartilhar o artigo os usuários não apenas participam de sua distribuição na mídia social, mas trazem uma efetiva contribuição para seu debate sinalizando que eles têm algo a dizer sobre o assunto.

\section{Considerações finais}

A pesquisa procurou contribuir com debate acerca dos estudos qualitativos no emergente campo da altmetria com vistas a compreensão dos contextos e condições de mensagens em mídias sociais sobre artigos científicos aplicando a análise de menções. Os resultados apontaram não haver muita disparidade entre os tipos de menções informativas (55,1\%) e conversacionais $(44,9 \%)$. Quanto as razões de menções, o ato de compartilhar supera as demais e representa 57,5\%. As razões de menções com comentário de cunho 
explicativo, narrativas exortativas, observações conclusivas e argumentativas somam 42,5\% e sinalizam não só o interesse, mas também, o debate em torno dos artigos.

Há uma preocupação dos autores deste trabalho quanto "aos rumos da altmetria" no que concerne ao aumento de pesquisas sobre as métricas alternativas, que este aumento não acabe por reproduzir o mesmo caminho trilhado pelas métricas tradicionais, de uma valorização exacerbada dos números, indicadores e fatores de impacto, descolados do diálogo dos contextos dos quais foram produzidos.

Para evitar esse caminho é necessário não perder de vista que, quando do seu recente surgimento em 2010, a altmetria clamava por um olhar do impacto social dos resultados de pesquisa, refletido sobretudo na atenção que desperta na sociedade no âmbito de uma ciência mais aberta e democrática.

Considera-se que, dado aos poucos estudos sobre o tema abordado torna-se necessário investir em propostas que considerem a análise das menções com vistas a entender as nuances da circulação da informação científica na web social e suas perspectivas. Tais estudos devem privilegiar as particularidades e dinâmicas que os ambientes digitais oferecem para a comunicação científica tendo em mente a natureza e as práticas desenvolvidas nas distintas fontes de dados, mídias sociais e por diferentes perfis de usuários, e assim compreender as relações entre as publicações e sua comunidade de atenção.

\section{Referências}

1. Silveira, M. A. A.; Caregnato, S. E. ; Bufrem, L. S. Estudo das razões das citações na Ciência da Informação: proposta de classificação. Tendências da Pesquisa Brasileira em Ciência da Informação [Internet]. 2014 [Citado em 13 ago. 2017]; 7; 232-250. Disponível em: http://inseer.ibict.br/ancib/index. $\mathrm{php/tpbci/article/view/163}$

2. Gouveia, F. C. A altmetria e a interface entre a ciência e a sociedade. Trabalho, Educação e Saúde, 2016 [Citado em 23 jul. 2017]; 14(3), 643-645. Disponível em: https://dx.doi.org/10.1590/1981-7746sip00126

3. Araujo, R. F.; Furnival, A. C. M. Comunicação científica e atenção online: em busca de colégios virtuais que sustentam métricas alternativas. Informação \& Informação (UEL. Online) [Internet]. 2016 [Citado em: 07 set. 2017 ]; 21: 68-89. Disponível em: http://dx.doi.org/10.5433/1981-8920.2016v21n2p68

4. Nelhans, G.; Lorentzen, D.G. Twitter conversation patterns related to research papers. Information Research, 2016 [Citado em 14 ago. 2017]; v.21, n.2, paper SM2. Disponível em: http://InformationR. net/ir/21-2/SM2.html

5. Costas, R.; Zahedi, Z.; Wouters, P. Do "altmetrics" correlate with citations? Extensive comparison of altmetric indicators with citations from a multidisciplinary perspective. Journal of the Association for Information Science and Technology [Internet]. 2014 [Citado em 28 ago. 2017]; 66 (10): 2003-2019. Disponível em: http://dx.doi.org/10.1002/asi.23309

6. Alperin, J. P. Exploring altmetrics in an emerging country context. ALTMETRICS14: EXPANDING IMPACTS AND METRICS; Bloomington, 2014. [Citado em 28 ago. 2017]. Disponivel em: http://dx.doi. org/10.6084/m9.figshare.1041797

7. Boon, C. Y.; Foon, J. W. J. Altmetrics is an indication of quality research or just hot topics. IATUL Conferences, 35th. Filand: Purdue University; 2014.

8. $\quad$ Fragoso, S.; Recuero, R.; Amaral, A. Métodos de pesquisa para internet. 1. ed. Porto Alegre: Sulina; 2011. 